\title{
Increasing exercise adherence among elderly patients with chronic disease in primary care: a prospective cohort study
}

\author{
Seth VanDerVeer ${ }^{1 *}$, Ronald Markert ${ }^{2}$, Brant Bickford ${ }^{1}$, Joseph Yuhas ${ }^{1}$, Paul Pikman ${ }^{1}$, Tim Wall ${ }^{1}$ and
} Kathryn Burtson ${ }^{1}$

\begin{abstract}
Background: Currently, the evidence for exercise in maintaining health, well-being, and physical functioning is overwhelming. Despite these benefits, more than $50 \%$ of the population fails to meet the recommended exercise requirements for age and health status. In our study, we sought to provide a method to increase exercise adherence that was both effective and time-efficient for physicians and their patients.

Methods: The primary objective of this research study was to evaluate the effectiveness of a graded exercise protocol and biweekly monitoring on increasing the duration of aerobic exercise to 150 min per week in a population of elderly individuals with chronic disease. Secondarily, we evaluated for improvement in resting heart rate, blood pressure, body mass index (BMI), and cardiorespiratory fitness. The overall study design was a randomized, prospective cohort study with assessor blinding. Forty-five patients aged $\geq 60$ years with multiple comorbidities were recruited from the Internal Medicine Clinic at Wright-Patterson AFB. Participants were randomized into a treatment or control arm and observed over a period of 34 weeks. Those in the treatment arm were given a graded walking protocol and received biweekly phone calls to evaluate compliance. Those in the control arm did not receive an intervention or biweekly monitoring. Measurements of heart rate, blood pressure, and BMI were taken quarterly in both groups. At the beginning and conclusion of the study, each participant completed a modified Balke treadmill test and Physical Activity Scale for the Elderly (PASE). Continuous variables were evaluated with the independent samples t-test, whereas categorical variables were evaluated with the chi-squared test.
\end{abstract}

Results: A greater percentage of the treatment group achieved the primary outcome (41.6\% vs. $0 \% ; p=0.003)$. Those in the treatment group also had favorable improvements in heart rate response $(-2.4$ beats $/ \mathrm{min} v \mathrm{vs} .+5.3$ beats/min; $p=0.038$ ) and PASE (+66 vs.-20; $p<0.001$ ). No significant differences were observed between groups for mean change in heart rate, blood pressure, or BMI.

Conclusion: Guided, independent exercise and surveillance can be an effective tool in primary care practice to help patients reach the recommended levels of exercise for both age and health status.

\footnotetext{
*Correspondence: sethvanderveer@gmail.com

${ }^{1}$ Wright Patterson Medical Center, USAF, Wright State University

Boonshoft School of Medicine, Dayton, USA
}

Full list of author information is available at the end of the article

\begin{abstract}
Introduction
There is an abundance of evidence supporting physical activity and physical fitness as effective in both lengthening and enhancing quality of life. Research has demonstrated that all body tissues and organ systems benefit from physical activity [1]. Despite these benefits, only a fraction of the population meets minimum exercise
\end{abstract}


recommendations, and the percentage decreases further as individuals age. In 2015, the Center for Disease Control and Prevention reported that less than $45 \%$ of elderly individuals aged $\geq 65$ years met the minimum aerobic exercise recommendations for age and health status; for those aged $\geq 85$ years, the minimum recommendation was achieved by less than $20 \%$. Within these groups, the lowest rates were seen among those with multiple comorbid conditions [2].

Multiple studies have shown that physicians are the preferred source of encouragement for exercise, and thus physicians are important in addressing the paucity of exercise compliance described above [3]. Exercise counseling by primary care physicians increases both interest and participation in exercise $[4,5]$. Despite this opportunity, many physicians do not counsel or monitor the physical activity behaviors of their patients [6]. Perceived barriers to exercise counseling and monitoring include lack of time, knowledge, training, and instruments or materials. Consequently, we sought to provide a solution that was convenient and understandable for patients as well as effective and time-efficient for physicians.

The primary objective of this study was to evaluate the effectiveness of a graded exercise protocol with biweekly (every 2 weeks) monitoring on increasing the duration of aerobic exercise to $150 \mathrm{~min}$ per week in a population of elderly individuals with chronic disease. Secondarily, we evaluated improvement in several health predictors including heart rate, blood pressure, body mass index (BMI), and cardiorespiratory fitness. It was hypothesized that the use of a graded exercise protocol with biweekly monitoring would be superior to usual care in increasing compliance with the minimum American College of Sports Medicine (ACSM) aerobic exercise recommendations for age and health status.

\section{Methods}

We performed a randomized cohort study with assessor blinding to evaluate the effect of a graded exercise protocol and biweekly monitoring on patient adherence to established exercise recommendations among elderly patients with chronic disease. The study was approved by the Wright Patterson AFB Medical Center (WPMC) Institutional Review Board (IRB). All participants provided written informed consent and HIPAA authorization. After a participant signed a HIPAA authorization, demographic and clinic data were retrieved from the outpatient clinic database.

Participants were recruited from Internal Medicine Clinic A at WPMC through print advertising and wordof-mouth. Inclusion criteria were: age $\geq 60$ years, ability to ambulate independently without the assistance of a walker, and ability to perform ambulation for $\geq 10 \mathrm{~min}$ at a slow pace on a level surface. All potentially eligible participants were invited to undergo an assessment of cardiorespiratory fitness with a modified Balke treadmill test. The modified Balke treadmill test was chosen for its safety and efficacy as a submaximal test of aerobic capacity in the elderly population [7]. The treadmill was set at $2.0 \mathrm{mph}$ with the grade starting at $0 \%$ and increasing by $2 \%$ every 3 min to a maximum of $10 \%$ over a total of 6 stages. Heart rate was recorded at 3-min intervals, which coincided with the conclusion of each stage. Subjects were excluded from the study if they failed to safely ambulate at $2.0 \mathrm{mph}$ during the modified Balke test.

A blinded statistician subsequently performed a computer-generated randomization sequence for subject allocation. Participants were randomly allocated to the treatment or control group and then scheduled for an appointment at Internal Medicine Clinic A. Baseline measurements of heart rate, blood pressure, and BMI were taken. Participants completed a Physical Activity Scale for the Elderly (PASE), which provided a qualitative assessment of baseline activity level. PASE is a comprehensive assessment of physical activity in the elderly, involving multiple domains ranging from recreational and work-related activities to household activities and caring for dependents. PASE has been validated in multiple studies over the past 20years [8,9].

Participants assigned to the treatment group received information and advice to complete an unsupervised, graded aerobic exercise protocol over a period of 34 weeks. The program featured walking at a low-intensity with the option of doing so on a treadmill at the gymnasium, at home, outside, or at another location such as a mall. Low-intensity activity was maintained through a self-administered talk test, which dictated that each participant was able to talk at all times during the activity. For each 2-week interval throughout the study, participants were given a designated walking time to complete. The program began with 2 days per week at 10 min per day and then progressed gradually. The number of exercise days was increased by 1 day every 8 weeks to a maximum of 5 days per week. The duration of daily exercise was increased by 1 min every 2 weeks until 22 min per day was achieved, and then by 2 min every 2 weeks to a maximum of 30 min per day.

No specific walking speed was prescribed since participants exhibited a wide range of baseline exertional capacity and potential for adaptive change. Table 1 shows that a greater increase in exercise activity was prescribed for the last 5 weeks since the participants were projected to have improved exercise capacity at this point. Table 1 outlines the graded aerobic exercise protocol. Participants in the treatment group were provided with a tracking sheet for their own accounting. They also received a short phone 
Table 1 Graded aerobic exercise protocol

\begin{tabular}{|c|c|}
\hline Week 1-2 & 2 DAYS, $10 \mathrm{~min}$ \\
\hline Week 3-4 & 2 DAYS, $11 \mathrm{~min}$ \\
\hline Week 5-6 & 2 DAYS, $12 \mathrm{~min}$ \\
\hline Week 7-8 & 2 DAYS, $13 \mathrm{~min}$ \\
\hline Week 9-10 & 3 DAYS, $14 \mathrm{~min}$ \\
\hline Week 11-12 & 3 DAYS, $15 \mathrm{~min}$ \\
\hline Week 13-14 & 3 DAYS, $16 \mathrm{~min}$ \\
\hline Week 15-16 & 3 DAYS, $17 \mathrm{~min}$ \\
\hline Week 17-18 & 4 DAYS, $18 \mathrm{~min}$ \\
\hline Week 19-20 & 4 DAYS, $19 \mathrm{~min}$ \\
\hline Week 21-22 & 4 DAYS, $20 \mathrm{~min}$ \\
\hline Week 23-24 & 4 DAYS, $21 \mathrm{~min}$ \\
\hline Week 25-26 & 5 DAYS, $22 \mathrm{~min}$ \\
\hline Week 27-28 & 5 DAYS, $24 \mathrm{~min}$ \\
\hline Week 29-30 & 5 DAYS, $26 \mathrm{~min}$ \\
\hline Week 31-32 & 5 DAYS, $28 \mathrm{~min}$ \\
\hline Week 33-34 & 5 DAYS, $30 \mathrm{~min}$ \\
\hline \multicolumn{2}{|c|}{ Initial - 2 days per week; 10 min per day } \\
\hline \multicolumn{2}{|c|}{ Days - Increase by 1 day every 8 weeks to a maximum of 5 days/week } \\
\hline \multicolumn{2}{|c|}{ Minutes - Increase by $1 \mathrm{~min}$ every 2 weeks to reach $30 \mathrm{~min} /$ day } \\
\hline
\end{tabular}

call every 2 weeks from a researcher to evaluate compliance. During each call, encouragement and confirmation of the designated walking time for the next 2-week interval were also provided.

Participants assigned to the control group did not receive the graded aerobic exercise protocol or biweekly phone calls from staff. Instead, participants were instructed to follow the advice of their primary care physician regarding aerobic exercise. Since most primary care physicians do not place sufficient emphasis on the importance of physical activity, they typically do not counsel patients beyond repeating the current exercise recommendations. In turn, patients are usually left without a clear plan to reach the recommended level of physical activity. Therefore, the exercise advice of the participant's own primary physician was selected to represent the standard of care for the control group.

In addition to the baseline assessments, both groups underwent quarterly vitals checks at week 12 and week 24 with repeat measurements of heart rate, blood pressure, and BMI. At the conclusion of the study, all patients underwent final testing, which included a vitals check, modified Balke treadmill test, and PASE. All testing followed a standard protocol.

The primary outcome was achievement of $150 \mathrm{~min}$ of aerobic exercise per week at the conclusion of the study. For those in the treatment group, exercise compliance was tracked biweekly as exercise duration was increased.
Exercise compliance was rated on a scale of 0 to $100 \%$ at $25 \%$ intervals. Achievement of $150 \mathrm{~min}$ of weekly aerobic exercise during the last 2 weeks of the graded aerobic exercise protocol (week 33 or week 34) was credited as $100 \%$ compliance. Those in the control group who did not or were unable to attend final testing were contacted by telephone and asked if they were walking at least 150 min per week.

Secondary outcomes included changes in PASE and heart rate response on the modified Balke treadmill test. Both were measured during the baseline and final testing periods to evaluate for changes in overall activity level and improvements in cardiorespiratory fitness. To evaluate for improvement in heart rate response on the modified Balke test, the heart rate change between Stage 1 and Stage 6 from the baseline test was compared to that of the final test. In addition, changes in heart rate, blood pressure, and BMI at vitals check were prespecified endpoints. Measurements of these values were completed at baseline, two quarterly checks, and final testing.

Due to the frequency and nature of data collection, all participants were aware of their placement in either the treatment or control group. Similarly, researchers involved in data tracking on compliance for the treatment group were aware of a participant's group placement. Additionally, the principal investigator, who participated in the measurement of vitals, modified Balke testing, and PASE, was aware of the group designation for each participant. However, WPMC technicians who participated in the baseline and first quarter vitals check were not aware of a participant's group designation. Further, the statistician, who performed all statistical analyses, was blinded to participant identity and group designation.

Data were entered into an Excel spreadsheet at WPMC. At the conclusion of the study, data were de-identified and sent to the statistician for analysis. Descriptive and inferential statistical analyses were performed with SPSS Statistics Version 25.0 (IBM, Armonk, NY) software. The chi-square test and independent samples t-test were used for group comparisons at an alpha level of $5 \%$.

Based on the assumption that the treatment group would have twice the percent as the control group achieving the primary outcome of $150 \mathrm{~min}$ of aerobic exercise per week at the conclusion of the study, a sample size of 25 in each group was estimated for statistical significance at alpha equal to 0.05 and with power of $80 \%$ (beta $=0.20$ ).

\section{Results}

Sixty-four volunteers were recruited from Internal Medicine Clinic A at WPMC in October 2019. All volunteers were screened with a modified Balke treadmill test. With 14 volunteers not appearing for the treadmill testing and 


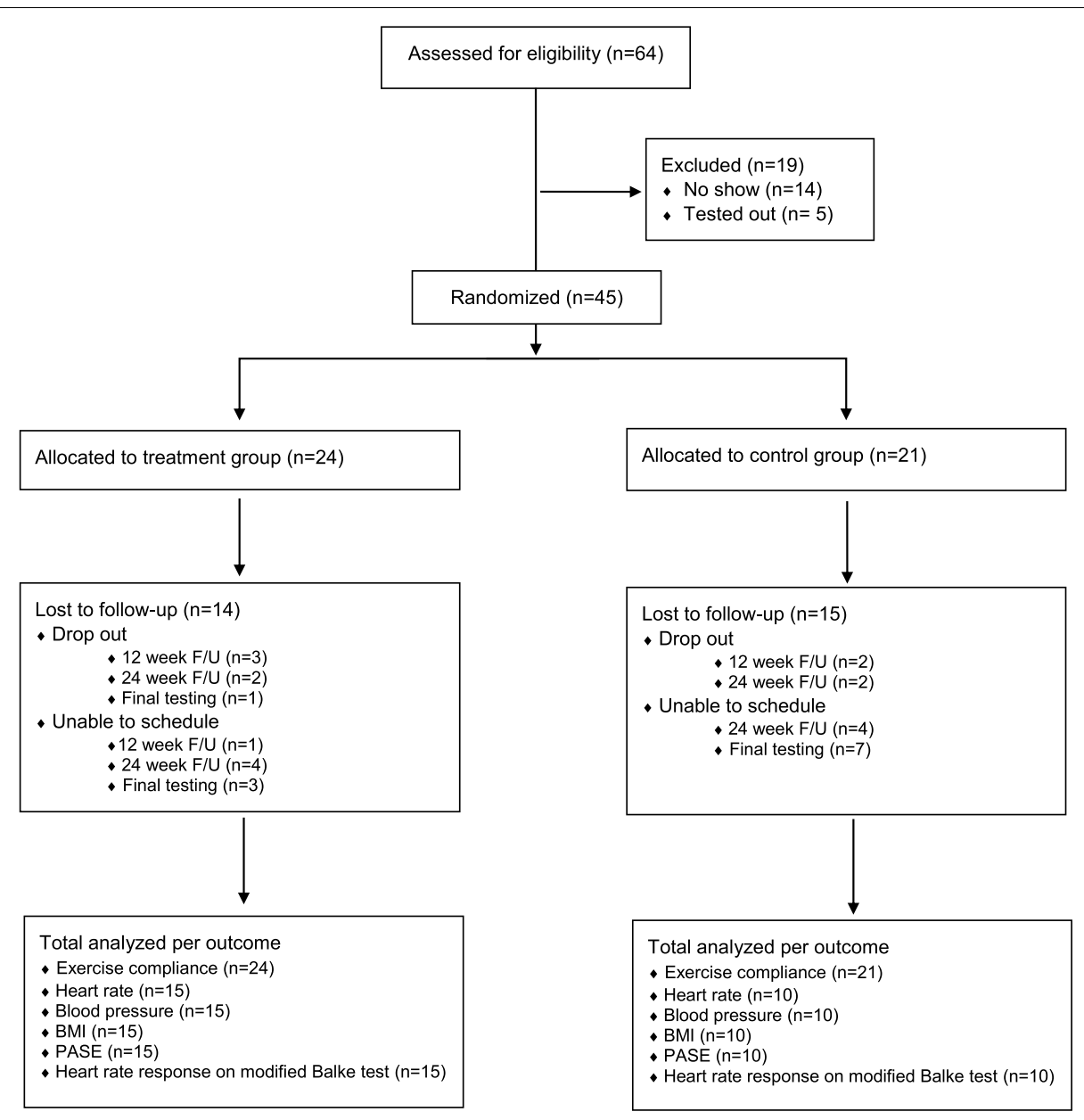

Fig. 1 Flow diagram of participation

5 volunteers unable to safely ambulate at $2.0 \mathrm{mph}, 45$ participants were eligible and agreed to participate. These participants were subsequently randomized to the exercise group $(n=24)$ and control group $(n=21)$. Figure 1 is a flow diagram of participation.

Table 2 shows that the baseline demographic and clinical characteristics were similar between the two groups. Follow-up at 12 weeks was completed by 39 of 45 participants (87\%), at 24 weeks by 27 of $45(60 \%)$, and at 34 weeks by 25 of 45 participants (55\%).

The treatment group $(n=24)$ had better exercise compliance with $41.7 \%$ of participants reaching the goal of $150 \mathrm{~min}$ of weekly aerobic exercise at 34 weeks compared to $0 \%$ in the control group $(n=21)(p=0.003)$ (Table 3$)$.

At 34 weeks, the treatment group $(n=15)$ had a mean increase in Physical Activity Score for the Elderly (PASE) of 66 points from baseline, whereas the control group $(n=10)$ had a mean decrease of 20 points $(p<0.001)$. For modified Balke testing, the treatment group had a mean decrease of 2.4 beats/min, whereas the control group had a mean increase in 5.3 beats $/ \mathrm{min}(p=0.038)$ (Table 3). No differences were observed between groups for mean change in heart rate $(p=0.06)$, systolic blood pressure $(p=0.49)$, diastolic blood pressure $(p=0.60)$, or body mass index $(p=0.65)$ (Table 3$)$.

\section{Discussion}

This 34-week, randomized cohort study with assessor blinding suggested that an unsupervised graded aerobic exercise protocol with biweekly monitoring is more effective than usual care for increasing exercise compliance among elderly individuals with chronic disease. Forty of our 45 participants had more than one comorbidity. Despite gym closures and limited access to exercise equipment during the COVID-19 pandemic, the treatment group had an impressive level of compliance with nearly $42 \%$ of participants achieving the goal of $150 \mathrm{~min}$ of weekly aerobic exercise. This finding is consistent with past studies demonstrating that patient participation in exercise was increased when physicians prescribed 
Table 2 Demographic and clinical characteristics for the treatment and control groups

\begin{tabular}{|c|c|c|c|}
\hline Demographic characteristics ${ }^{a b}$ & $\begin{array}{l}\text { Treatment } \\
n=24\end{array}$ & $\begin{array}{l}\text { Control } \\
n=21\end{array}$ & $P \neq$ \\
\hline Age, $y r-m e a n \pm s d$ & $72.5 \pm 7.2$ & $73.3 \pm 8.2$ & 0.35 \\
\hline Male, $n(\%)$ & $17(70.8)$ & $10(47.6)$ & 0.11 \\
\hline Caucasian, $n(\%)$ & $21(87.5)$ & $19(90.5)$ & 1.00 \\
\hline \multicolumn{4}{|l|}{ Clinical characteristics $^{b}$} \\
\hline Weight, lb. - mean \pm sd & $208 \pm 45.4$ & $192.3 \pm 45.6$ & 0.26 \\
\hline $\mathrm{BMI}-\operatorname{mean} \pm s \mathrm{~d}$ & $31.4 \pm 5.5$ & $31.0 \pm 6.8$ & 0.84 \\
\hline Resting heart rate, bpm - mean $\pm s d$ & $72.8 \pm 11.8$ & $72.5 \pm 10.1$ & 0.92 \\
\hline Systolic blood pressure, $\mathrm{mmHg}-$ mean \pm sd & $135.8 \pm 15.8$ & $133.0 \pm 18.8$ & 0.59 \\
\hline Diastolic blood pressure, $\mathrm{mmHg}-$ mean \pm sd & $79.1 \pm 8.5$ & $76.0 \pm 8.3$ & 0.20 \\
\hline Physical Activity Score for the Elderly - mean \pm sd & $123.1 \pm 46.6$ & $132.4 \pm 47.1$ & 0.51 \\
\hline Obesity, n(\%) & $16(66.7)$ & $11(52.4)$ & 0.33 \\
\hline Diabetes mellitus, $n(\%)$ & $12(50.0)$ & $6(28.6)$ & 0.14 \\
\hline Hypertension, $n(\%)$ & $20(83.3)$ & $16(76.2)$ & 0.82 \\
\hline Dyslipidemia, n(\%) & $16(66.7)$ & $15(71.4)$ & 0.73 \\
\hline Obstructive sleep apnea, n(\%) & $6(25.0)$ & $7(33.3)$ & 0.54 \\
\hline Atrial fibrillation/flutter, $n(\%)$ & $7(29.2)$ & $1(4.8)$ & 0.08 \\
\hline Congestive heart failure, $n(\%)$ & $1(4.2)$ & $1(4.8)$ & 1.00 \\
\hline Coronary artery disease, $n(\%)$ & $5(20.8)$ & $3(14.3)$ & 0.86 \\
\hline Peripheral vascular disease, $n(\%)$ & $1(4.2)$ & 0 & 1.00 \\
\hline Stroke, $n(\%)$ & $1(4.2)$ & $1(4.8)$ & 1.00 \\
\hline Chronic kidney disease, $n(\%)$ & $4(16.7)$ & $5(0.238)$ & 0.82 \\
\hline Asthma, $n(\%)$ & $1(4.2)$ & $1(4.8)$ & 1.00 \\
\hline Chronic obstructive pulmonary disease, $n(\%)$ & $1(4.2)$ & $2(9.5)$ & 0.91 \\
\hline Anemia, $n(\%)$ & $1(4.2)$ & 0 & 1.00 \\
\hline Malignancy, $n(\%)$ & $2(8.3)$ & $1(4.8)$ & 1.00 \\
\hline Hypothyroidism, n(\%) & 0 & $3(14.3)$ & 0.19 \\
\hline Hyperthyroidism, n(\%) & 0 & $1(4.8)$ & 0.95 \\
\hline Kleinfelter syndrome, $n(\%)$ & $1(4.2)$ & 0 & 1.00 \\
\hline Osteoporosis, $n(\%)$ & $2(8.3)$ & $2(9.5)$ & 1.00 \\
\hline Osteoarthritis, $n(\%)$ & $7(29.2)$ & $7(33.3)$ & 0.76 \\
\hline Rheumatoid arthritis, n(\%) & 0 & $1(4.8)$ & 0.95 \\
\hline Fibromyalgia, $n(\%)$ & 0 & $1(4.8)$ & 0.95 \\
\hline Degenerative disc disease, $n(\%)$ & $4(16.7)$ & $5(23.8)$ & 0.82 \\
\hline Spinal stenosis, $n(\%)$ & $1(4.2)$ & $2(9.5)$ & 0.91 \\
\hline Depression, $n(\%)$ & $1(4.2)$ & $3(14.3)$ & 0.51 \\
\hline
\end{tabular}

sd Standard deviation

${ }^{a}$ All study participants lived either independently or with a spouse. ${ }^{b}$ Continuous variables are reported as mean \pm standard deviation; categorical variables are reported as count (percentage). $¥$ Independent samples $t$ test for continuous variables; chi-squared test for categorical variables

specific recommendations $[4,5]$. Further, this result was achieved in a population with arguably the most physical and emotional obstacles to exercise and physiologic adaptation. Unhappily, the study began just prior to the arrival of COVID-19 in southwest Ohio, and the pandemic may well have lessened the effectiveness of the program. Nevertheless, our principal finding reinforces the importance of physicians assuming a more active role in promoting physical activity for their patients.
Participants in the treatment group benefited with respect to PASE and heart rate response on the modified Balke treadmill test. The PASE improvement reflected an increase in physical activities such as recreation, housework, home repairs, yard care, outdoor gardening, and volunteer work. The PASE improvement suggests that the protocol not only motivated more exercise, but also created an expectation for exercise to become a necessary part of one's daily routine. In a 2019 study, a higher 
Table 3 Comparison of treatment group and control group on outcomes

\begin{tabular}{|c|c|c|c|}
\hline Outcome & Treatment Group & Control Group & $P^{*}$ \\
\hline 150 min of weekly aerobic exercise $-n(\%)^{1}$ & $10(41.7)$ & $0(0.0)$ & 0.003 \\
\hline \multicolumn{4}{|l|}{ Physical Activity Scale for the Elderly (0 to 400 ) } \\
\hline Change: baseline to 34 weeks $^{2}$ & increase 66 & decrease 20 & $<0.001$ \\
\hline \multicolumn{4}{|l|}{ Balke heart rate: change from Stage 1 to 6 - bpm } \\
\hline Change: baseline to 34 weeks ${ }^{2}$ & decrease 2.4 & increase 5.3 & 0.038 \\
\hline \multicolumn{4}{|l|}{ Vitals check: heart rate } \\
\hline Change: baseline to 34 weeks $^{2}$ & increase 6.9 & increase 0.8 & 0.06 \\
\hline \multicolumn{4}{|l|}{ Vitals check: systolic blood pressure } \\
\hline Change: baseline to 34 weeks $^{2}$ & decrease 5.9 & decrease 0.3 & 0.49 \\
\hline \multicolumn{4}{|l|}{ Vitals check: diastolic blood pressure } \\
\hline Change: baseline to 34 weeks ${ }^{2}$ & decrease 0.4 & increase 1.5 & 0.60 \\
\hline \multicolumn{4}{|l|}{ Vitals check: body mass index } \\
\hline Change: baseline to 34 weeks $^{2}$ & decrease 0.16 & increase 0.11 & 0.65 \\
\hline
\end{tabular}

PASE was related to increased strength and muscle mass in elderly adults and was protective against frailty and sarcopenia [9]. Perhaps, most importantly, the improvement in the final PASE, taken in the midst of the COVID19 pandemic, speaks to the consistency that our protocol fostered. Not only did participants in the treatment group improve their activity levels, but they were able to achieve peak conditioning when the barriers to exercise were the greatest. Similar to the increased PASE in the treatment group, the improvement in heart rate response on the modified Balke test demonstrated enhanced cardiorespiratory fitness, which has been found to reduces rates of all-cause and disease-specific mortality in multiple studies over the past 30years [10]. Despite the exercise protocol being limited to low-intensity activity, participants were able to benefit from an increase in aerobic conditioning.

While the Balke change in heart rate from baseline to week 34 favored the treatment group over the control group by nearly $8 \mathrm{bpm}(-2.4 \mathrm{bpm}$ vs. $+5.3 \mathrm{bpm})$, heart rate change from baseline to week 34 with the vitals check favored the control group over the treatment group by over $6 \mathrm{bpm}(+0.8 \mathrm{bpm}$ vs. $+6.9 \mathrm{bpm})$. This conflict in results was likely due to a deviation in protocol caused by lack of resources and time restrictions. Both treatment and control group participants were not allowed to wait the usual 5-min period to relax prior to measurement of their vitals during final testing. This deviation from the protocol may have led to artificially elevated heart rates that were not true representations of the participants' cardiorespiratory fitness. We are obligated to report all findings, but believe the Balke measurements of heart rate were the more trustworthy.

Blood pressure change favored the treatment group over the control group (SBP: -5.9 vs. -0.3 and DBP -0.4 vs. +1.5$)$, but our study's small sample size precluded these differences of $5.6 \mathrm{mmHg}$ for SBP and $1.9 \mathrm{mmHg}$ for DBP from being statistically significant. Also, COVID-19 restrictions resulted in different $\mathrm{BP}$ measuring devices being used at baseline, quarterly, and final measurements. Finally, BMI change also favored the treatment group $(-0.16$ vs. +0.11$)$. This small $0.27 \mathrm{BMI}$ benefit for the treatment group demonstrates the relative immutability of weight reduction in elderly adults when only 150 minutes of weekly aerobic exercise at a low intensity is prescribed.

To our knowledge, this study was the first to specifically evaluate the effect of a graded exercise protocol and biweekly monitoring on exercise compliance in elderly individuals with multiple comorbidities. Though numerous studies have evaluated the effectiveness of many different exercise protocols in various populations, patient compliance remains the principal barrier to exercise in the primary care setting. We developed a patient-centered approach to exercise that addresses many of the reported obstacles to exercise counseling and provides a time-efficient method that can be applied readily to outpatient practice. The notable improvements in patient adherence occurred with only a minimal time investment from physicians. We used only a single exercise handout in combination with a short biweekly phone call, both of which were simple and effective. Importantly, the 
simplicity of our approach lends itself to implementation by other members of the medical team to include nurses, medical assistants, or other support staff, which may be the best option for integration into a busy primary care practice. In addition, our program achieved favorable results in a difficult population of patients, many of whom had severe lack of cardiopulmonary reserve. Consequently, a similar approach may be even more effective in healthier populations with fewer barriers to exercise and more ability to adapt physiologically.

Though practical constraints limited our study to 34 weeks, it is feasible to use an exercise protocol with a similar rate of change over a much longer period. Once patient adherence is established, a similar model can be applied to greater exercise duration or used to maintain a patient's current fitness level and preserve their functionality for years to come. Ideally, there would be regular follow-up appointments throughout the program to examine future goals. As mentioned previously, these follow-up appointments could be performed by other members of the medical team (nurse, medical assistant, etc.) instead of the physician, unless the exercise prescription requires significant modification.

The most notable limitation of our study was COVID19. The arrival of the pandemic led to an increase in the dropout rate for both groups, which limited the power of our study. Exercise compliance among those in the treatment group also decreased due to gym and mall closures as well as fear of leaving the house. Furthermore, the pandemic impacted our planned consistency for participant measurement. Prior to COVID-19, our plan was to perform all baseline, quarterly, and final measurements of heart rate, blood pressure, and BMI in our clinic with the same staff, protocols, and measuring devices. Similarly, we planned to perform the baseline and final modified Balke tests at the AFB's gym on the same treadmill. However, when the base clinics and gym were closed, these measurements had to be performed at alternative locations and with unstandardized equipment having unknown calibrations. Consequently, measurement error was likely increased due to these unanticipated disruptions. Furthermore, limited hours of operation and equipment access during the pandemic prevented many participants from completing final testing. The chain of events caused by the COVID-19 pandemic also affected our pre-planned sample size designed to assure that clinically meaningful differences were also statistically significant. In addition, the study was conducted at a single military treatment facility. Thus, generalizability to other settings should be done with caution. Lastly, we acknowledge that our study design allowed for recall bias since it relied heavily on accurate reporting from the participants.
In conclusion, our graded aerobic exercise protocol with biweekly monitoring led to greater improvements in exercise compliance, PASE, and cardiorespiratory fitness when compared with usual care at 34 weeks in a population of elderly individuals with chronic disease. We believe that guided, independent exercise and surveillance can be an effective tool in primary care practice to help patients reach the recommended levels of exercise for both age and health status. However, given the small sample size in our study, future studies in elderly individuals with chronic disease are needed to confirm these findings. Future studies utilizing a team approach to patient exercise programming and surveillance with the possible addition of a fitness professional may lead to even greater changes in patient adherence and are strongly encouraged.

\section{Acknowledgements}

Airman Devin Figures for his assistance with participant recruitment.

\section{Authors' contributions}

SV created the study protocol, submitted the IRB, assigned roles to the other researchers, recruited all participants, performed all baseline and final testing, collected data on a designated group of participants in the treatment and control group for the duration of the study, served as the primary author of the manuscript, and participated in all edits. RM advised on all steps of the research process, performed all statistical analyses on the study data, authored the statistical portions of the manuscript, and edited all versions of the manuscript and other supplementary materials. BB and JY collected data on a designated group of participants in the treatment and control group for the duration of the study and participated in final edits. PP and TW collected data on a designated group of participants in the treatment and control group for the duration of the study and reviewed the manuscript. KB served as the main research advisor and overseer of the study, and contributed to edits on all versions of the manuscript and supplementary materials. The author(s) read and approved the final manuscript.

\section{Funding}

None.

Availability of data and materials

All data generated or analysed during this study in this published article and its supplementary information files.

\section{Declarations}

\section{Ethics approval and consent to participate}

This study was approved via expedited review by the Wright Patterson Medical Center (WPMC) Institutional Review Board (IRB) on 8/30/19. The assigned reference number for the protocol is FWP20190004H. Risk level was determined to be Minimal Risk 32 CFR 219. The associated assurance number was DoD Assurance F50005 / FWA 00000609 / IRB 00001357. In accordance with our protocol, written informed consent and HIPAA authorizations were completed by all participants. All methods were carried out in accordance with relevant guidelines and regulations.

\section{Consent for publication}

Not applicable.

Competing interests

The authors declare that they have no competing interests. 


\section{Author details}

'Wright Patterson Medical Center, USAF, Wright State University Boonshoft School of Medicine, Dayton, USA. ${ }^{2}$ Wright State University Boonshoft School of Medicine, Dayton, USA.

Received: 12 April 2021 Accepted: 27 September 2021

Published online: 01 November 2021

\section{References}

1. Moore GE, Durstine JL, Painter PL. ACSM's exercise Management for Persons with chronic diseases and disabilities. 4th ed. Champaign: Human Kinetics, Inc.; 2016.

2. QuickStats. Percentage of Adults Aged $\geq 65$ Years Meeting 2008 Federal Guidelines for Leisure-Time Aerobic and Muscle-Strengthening Activities, by Age and Type of Activity - United States, 2000-2002 and 2013-2015. MMWR Morb Mortal Wkly Rep. 2016;65:1019.

3. Petrella RJ, Lattanzio CN. Does counseling help patients get active? Can Fam Physician. 2002:48:72-9.

4. Crookham J. A guide to exercise prescription. Prim Care. 2013;40:801-20
5. Weidinger KA, Lovegreen SL, Elliott MB, et al. How to make exercise counseling more effective: lessons from rural America. J Fam Pract. 2008;57:394-402

6. Petrella RJ, Koval JJ, Cunningham DA, et al. Can primary care doctors prescribe exercise to improve fitness? Am J Prev Med. 2003:24:316-22

7. Hagberg JM. Exercise assessment of arthritic and elderly individuals. Baillière's Clin Rheumatol. 1994;8:29-52.

8. Martin KA, Rejeski WJ, Miller ME, et al. Validation of the PASE in older adults with knee pain and physical disability. Med Sci Sports Exerc. 1999;31:627-33.

9. Curcio F, Liguori I, Cellulare M, et al. Physical activity scale for the elderly (PASE) score is related to sarcopenia in noninstitutionalized older adults. J Geriatr Phys Ther. 2019;42:130-5.

10. Harber MP, Kaminsky LA, Arena R, et al. Impact of cardiorespiratory fitness on all-cause and disease-specific mortality: advances since. Prog Cardiovasc Dis. 2009;60:11-20.

\section{Publisher's Note}

Springer Nature remains neutral with regard to jurisdictional claims in published maps and institutional affiliations.
Ready to submit your research? Choose BMC and benefit from:

- fast, convenient online submission

- thorough peer review by experienced researchers in your field

- rapid publication on acceptance

- support for research data, including large and complex data types

- gold Open Access which fosters wider collaboration and increased citations

- maximum visibility for your research: over $100 \mathrm{M}$ website views per year

At BMC, research is always in progress.

Learn more biomedcentral.com/submissions 